\title{
Les changements politiques en Espagne après
}

\section{Brumaire}

\section{Emilio La Parra López}

\section{(2) OpenEdition \\ 1 Journals}

\section{Édition électronique}

URL : https://journals.openedition.org/ahrf/295

DOI : $10.4000 /$ ahrf.295

ISSN : 1952-403X

Éditeur :

Armand Colin, Société des études robespierristes

\section{Édition imprimée}

Date de publication : 1 décembre 1999

Pagination : 695-712

ISSN : 0003-4436

\section{Référence électronique}

Emilio La Parra López, "Les changements politiques en Espagne après Brumaire », Annales historiques de la Révolution française [En ligne], 318 | octobre-décembre 1999, mis en ligne le 11 avril 2006, consulté le 23 avril 2022. URL : http://journals.openedition.org/ahrf/295 ; DOI : https://doi.org/ 10.4000/ahrf.295

Ce document a été généré automatiquement le 23 avril 2022.

Tous droits réservés 


\title{
Les changements politiques en Espagne après Brumaire
}

\author{
Emilio La Parra López
}

1 Brumaire coïncida avec une conjoncture politique interne spécialement critique en Espagne. Le gouvernement, dirigé par Mariano Luis de Urquijo ${ }^{1}$, s'était engagé dans une ambitieuse politique réformiste qui touchait directement le pouvoir de l'Inquisition et de l'Église. De 1798 à 1800 on décréta la desamortización d'une grande partie des biens des institutions fortement liées à l'Église, on attaqua le pouvoir de l'Inquisition, en arrivant même à destituer tous les membres du tribunal de Barcelone, et on commença à établir les bases pour construire une Église nationale, dirigée par les évêques et relativement indépendante de Rome du point de vue économique et juridique ${ }^{2}$ À l'intérieur du pays, le gouvernement comptait pour le développement de cette politique sur la collaboration d'un groupe, réduit mais influent, d'illustrés, connu à l'époque comme el partido jansenista ; à l'extérieur, Urquijo croyait pouvoir compter sur le Directoire et la décadence du pouvoir de la curie romaine, à cause des difficiles relations entre celle-ci et la France et des difficultés personnelles de Pie VI ${ }^{3}$. En revanche, un groupe, important et hétérogène, composé de la hiérarchie ecclésiastique, l'envoyé du pape (dont les fonctions étaient pratiquement suspendues), l'Inquisition et de nombreux courtisans, se constitua face au gouvernement, à cause de son refus de l'alliance signée avec la France en 1796. Tous ceux-ci comptaient sur l'aide des groupes de pression, constitués par des aristocrates et soutenus par l'Angleterre et Naples, et ils étaient connus sous le nom de el partido inglés o italiano. En tout cas, ce qui fut réellement déterminant, ce fut l'alignement sur ce front d'opposition de Manuel Godoy et de la reine Marie-Louise, tous deux mécontents d'avoir perdu une partie de leur pouvoir personnel, et non pas vraiment l'orientation politique du gouvernement.

2 Lors du coup d'État de Brumaire, la conjoncture économique espagnole était déplorable : d'énormes difficultés entravant le commerce et le maintien des relations avec l'Amérique, les considérables dépenses qui résultaient de l'alliance avec la France et les affrontements permanents avec l'Angleterre, en étaient la cause. Malgré tout, c'était la lutte interne qui préoccupait surtout les cercles du pouvoir, avec toutes sortes 
d'intrigues et de manœuvres entre les deux groupes, dirigés respectivement par Urquijo et Godoy. Le revirement politique de la France après Brumaire affecta directement cette situation, car il fut interprété en Espagne de telle façon qu'il créa un climat propice pour le triomphe des opposants à Urquijo, sinon dans l'immédiat, du moins une année après l'instauration du Consulat. Cela prouve que, contrairement à ce qui arriva à l'époque du Directoire ${ }^{4}$, il n'y eut en cette circonstance aucune intervention directe du nouveau régime français sur le changement politique interne espagnol, bien qu'après Brumaire il fut évident qu'il était impossible de continuer la politique suivie par Urquijo. Néanmoins, en politique extérieure, et comme chacun le sait, l'influence du Consulat, ou plus exactement de Bonaparte, fut directe et déterminante. Dans ce domaine, Brumaire créa de nouvelles incertitudes sur la position internationale de l'Espagne, rendant plus difficiles ses relations avec la France et accroissant son engagement international. Ceci provoqua certaines innovations sans altérer fondamentalement l'orientation de la politique extérieure espagnole, déterminée par la dépendance vis-à-vis de la France et par l'affrontement avec l'Angleterre.

Accueil de la nouvelle du coup d'État

3 Le coup d'État du 18 Brumaire fut, en général, bien accueilli dans les milieux officiels espagnols, bien que quelques personnages décisifs, comme la reine et Godoy, exprimèrent des préventions dans leur correspondance personnelle. La nouvelle de la fin du Directoire fut un soulagement pour la majorité des Espagnols, car le régime était détesté à cause de ses interventions dans les affaires internes et de ses exigences continues, et parfois contradictoires, dans l'application du traité d'alliance signé en 1796. Les relations diplomatiques avec le Directoire s'étaient détériorées considérablement depuis 1798 et, de plus, il existait en Espagne la conviction généralisée que le gouvernement français encourageait des projets afin d'étendre la révolution au territoire du monarque espagnol et d'en finir même avec la monarchie.

4 Les premières informations sur le coup d'État offertes - parfois avec enthousiasme par l'ambassadeur espagnol à Paris, Ignacio Múzquiz, ne purent en aucun cas être plus positives. La première communication envoyée après Brumaire, le 11 novembre, relate en détail les faits et souligne l'importance de l'événement: le nouveau régime " cambiará enteramente la constitución de la Francia y la política de toda Europa " ${ }^{5}$. Il expose ensuite trois caractéristiques sur lesquelles il insistera dans des correspondances postérieures : l'accueil favorable de l'opinion publique française, les perspectives ouvertes pour obtenir la paix en Europe et la capacité du nouveau régime pour obtenir la pacification politique en France ${ }^{6}$. Ensuite, il livre des informations sur les premières décisions du Consulat, en particulier sur le bon choix quant à la nomination du nouveau gouvernement et du personnel des ministères ${ }^{7}$, ainsi que sur l'opportunité de la nouvelle Constitution, dont il souligne le renforcement du pouvoir exécutif, grâce auquel « podrá producir muy buenos efectos y desde luego contendrá las insurrecciones del interior ${ }^{8}$. Selon Múzquiz, le Consulat avait débuté de la meilleure façon, grâce à « las reformas saludables que se van haciendo en todos los ramos de la administración, que producen el mejor efecto y así va adquiriendo cada día el nuevo Gobierno la opinión que justamente se merece. ${ }^{9}$

5 Le caractère du coup d'État de Brumaire ne surprit, en aucun cas, ni Múzquiz, ni la Cour espagnole qu'il avait déjà renseignée sur les agissements de Bonaparte et sur l'excellent accueil qu'il avait reçu en France à son retour d'Égypte. L'ambassadeur espagnol suivit 
attentivement la trajectoire politique du général depuis son débarquement à Fréjus et, à tous moments, il en transmit à son gouvernement une image favorable. Dans les jours qui suivirent le coup d'État, il écrivait : "No se habla por ahora de darle algún mando en el ejército, pero es regular que quieran en adelante sacar partido del ascendiente que tiene sobre los demás generales y sobre los franceses $»^{10}$. Cette appréciation de l'ambassadeur ne provenait pas tant de sa perspicacité politique que de l'information dont il disposait, car Múzquiz maintenait d'excellentes relations avec Sieyès depuis leur rencontre à Berlin ${ }^{11}$, où ils avaient occupé tous deux un poste diplomatique. Pour une raison ou une autre, leurs rapports contribuèrent à créer sans aucun doute à la Cour espagnole une prédisposition favorable à l'égard du nouveau régime français.

6 Par ailleurs, Urquijo reçut à titre personnel d'autres impressions de Paris. Son ami et confident Pedro Gómez Labrador, employé à l'ambassade espagnole, lui écrivit le 18 novembre une lettre personnelle où il ne présageait rien de bon pour ce nouveau régime : «Cuente V. que antes de ventôse (sic) la mitad de Francia dé al diablo con la revolución de las ballonetas. ${ }^{12}$. Il justifiait ses prédictions par les nouvelles mesures fiscales et les difficultés pour obtenir immédiatement la paix en Europe, avec lesquelles le gouvernement avait voulu éblouir la foule. D'un ton intime et aimable, et même sincère, Urquijo lui répondit : «Amigo Perico : pienso como VM en punto a ese país y me ha gustado mucho su carta del 18. ${ }^{13}$

7 En effet, Urquijo n'accueillit pas favorablement le coup d'État de Brumaire ${ }^{14}$ et reprocha à Múzquiz son enthousiasme dans ses dépêches. Un an après le coup d'État, Múzquiz est encore obligé de se défendre contre les reproches du ministre en lui adressant une lettre autographe et confidentielle: "Los miramientos que tengo a estas gentes los tengo no por ello sino por el bien de los negocios; repito que ni los estimo ni los quiero ni los temo y que sólo deseo dejarlos cuanto pueda " ${ }^{15}$. Quant au gouvernement français, il remarque qu'il est "Casi todavía revolucionario, en cuyos individuos no falta soberbia, violencia, poca estabilidad y vanagloria de sus triunfos » ${ }^{16}$ et il conclut en insistant sur son désir d'abandonner l'ambassade de Paris, « donde no tengo más que disgustos " ${ }^{17}$. Múzquiz fut sûrement sincère en écrivant ces mots, de la même façon qu'il le fut auparavant en exposant sa vision positive des espérances créées par Brumaire. Son attitude envers la France, d'une certaine manière schizofrène où continuellement la méfiance et l'admiration se mêlaient, fut caractéristique des gouvernants espagnols de la période révolutionnaire. Brumaire ne pouvait l'éliminer complètement, malgré l'impression générale du revirement conservateur du nouveau régime.

8 L'impression positive sur la signification de Brumaire transmise par Múzquiz fut corroborée par l'important groupe d'émigrés français à qui des correspondants locaux en France avaient transmis les rumeurs les plus variées sur le changement d'attitude du nouveau gouvernement et la suppression des listes. Parmi eux s'affirma la conviction que le nouveau régime mettrait fin à la Révolution, assumerait avec intérêt l'ouverture de conversations pour l'établissement de la paix en Europe et qu'il résoudrait la confuse situation interne de la France grâce à la consolidation du pouvoir exécutif. Ceci était particulièrement intéressant pour la Cour espagnole car, en cas de succès, cela permettrait de destiner les troupes occupées à lutter contre la Contre-Révolution à la consolidation du pouvoir français en Italie. L'agrandissement du duché de Parme, si convoité par la reine Marie-Louise et ligne maitresse de l'activité extérieure de l'Espagne, pourrait, par conséquent, s'accomplir ainsi. Múzquiz, conscient de tout cela, 
insista spécialement dans ses rapports sur la condamnation du soulèvement de la chouannerie, se référant aux progrès obtenus depuis l'installation du nouveau régime. Son opinion à ce sujet, émise dans plusieurs dépêches, est parfaitement résumée dans celle du 21 février 1800 : «La pacificación de los chuanes (sic) continúa haciendo los progresos más rápidos... y apenas se duda que veamos bien pronto la feliz conclusión de guerra tan funesta. ${ }^{18}$

9 La Cour espagnole attendait du nouveau régime non seulement l'aide nécessaire pour accomplir finalement ses désirs italiens, mais aussi quelque chose de plus important : la fin de la Révolution en France et, en conséquence, la fin des tentatives pour l'exporter en Espagne. Cette idée marqua Charles IV profondément. Selon Godoy, le roi accueillit favorablement l'avènement du Consulat "por la enemistad abierta que mostraba el primer cónsul contra las ideas y pasiones demagógicas " ${ }^{19}$ et influencé par certaines opinions, qui provenaient spécialement des émigrés, il en arriva à espérer que Bonaparte rétablisse une monarchie constitutionnelle ${ }^{20}$. Cette impression eut une certaine répercussion dans les milieux espagnols à en juger par les notes du jésuite Luengo, immédiatement après avoir reçu la nouvelle de Brumaire : « ...ha caído por tierra la Gran República Democrática filosófica de Francia y se ha acabado el gobierno propiamente popular y democrático y empieza un gobierno aristocrático y casi monárquico " ${ }^{21}$. L'idée persiste durant un certain temps et en avril 1801 Lucien Bonaparte la transmet à son frère, la présentant comme une synthèse du sentiment général des Espagnols ${ }^{22}$.

10 Le roi d'Espagne fut influencé sans aucun doute par les idées de Nicolás de Azara, un personnage très influent à ce moment-là à la Cour et qui connaissait profondément la France, puisqu'il venait de quitter le poste d'ambassadeur à Paris. Azara était ami de Bonaparte. Ils s'étaient rencontrés et avaient négocié ensemble en Italie quelques années auparavant et ils avaient eu une longue conversation le 17 octobre 1799, soit le lendemain de l'arrivée du général à Paris. Ennemi déclaré d'Urquijo et du Directoire, Azara put sans doute transmettre au roi et à la reine, ainsi qu'à Godoy (très proche de lui à l'époque), une impression favorable sur le nouvel homme fort de la France.

11 La personnalité de Bonaparte suscitait dans les cercles de pouvoir espagnols à la fois un sentiment de crainte et d'espoir. Tous admiraient sa capacité militaire et croyaient qu'une fois consolidé, son pouvoir politique en France, il obtiendrait le contrôle de l'Europe. C'est à cause de cela que les groupes espagnols opposés à l'alliance avec la France furent très déçus, car ils perdirent leur confiance en une victoire britannique et, par conséquent, leur position politique s'affaiblit à l'intérieur du pays. Cependant, à la Cour on y vit avec espoir la possibilité d'arriver à la paix en Europe (aspect important étant donné la situation économique de la monarchie) et d'accomplir les projets espagnols en Italie. Ce dernier désir fut confirmé tout de suite par la campagne victorieuse de 1800. Comme on pouvait l'imaginer, Bonaparte mit hors jeu l'Autriche, opposant principal aux projets espagnols d'agrandissement du duché de Parme et, en conséquence, offrit au roi et à la reine les meilleures perspectives d'avenir. Charles IV, moins ingénu qu'on ne le présentait, profita de l'occasion pour vanter les mérites du Premier Consul devant l'ambassadeur français Alquier. Ainsi que celui-ci le fit savoir à Talleyrand, une fois reçue la nouvelle de la bataille de Marengo, le roi lui avait dit: «Bonaparte no tiene un amigo más franco y leal que Carlos IV... Sabe tan bien gobernar como batirse... Es un gran hombre al que amo de todo corazón; y tan buen católico como yo! ${ }^{23}$ 

Brumaire et le rapprochement immédiat de Bonaparte et de l'Église catholique redonna des espérances au groupe formé autour de la reine et de Godoy et affaiblit sérieusement la position d'Urquijo, ainsi que le front adverse des partisans du rapprochement avec l'Angleterre. Brumaire créa donc un nouveau climat favorable au changement politique en Espagne.

Changement politique en Espagne

Bonaparte avait confiance en l'utilité matérielle de l'alliance avec l'Espagne, bien que comme l'a signalé Fugier ${ }^{24}$, il était mal informé de la situation réelle de la monarchie. Comme la majorité des Français de l'époque, il surestimait les possibilités de son armée, dont il attendait bien plus qu'elle ne pouvait faire, ainsi que la capacité d'influence de la famille royale en Italie et il se fit des illusions sur les réserves de métaux précieux en Amérique, mais en même temps il méprisait le pays à cause de son retard, qu'il attribuait à l'Inquisition et au fanatisme. Il jugeait la capacité politique des gouvernants espagnols avec autant de mépris. Bonaparte était convaincu de leur incapacité et de la prépondérance de l'intrigue et de la corruption. Cette idée le poursuivit longtemps et il agit en conséquence dans ses relations diplomatiques avec l'Espagne. Dans les instructions données à Alquier en l'envoyant à l'ambassade à Madrid, il présente très clairement son point de vue : «l'esprit de versatilité qui paraît dominer dans le Conseil $\mathrm{du}$ roi d'Espagne, les intrigues qui agitent ses plus intimes entourages, les violentes animosités qui un jour semblent se tourner en moyens de bonne intelligence et en éléments de coalition, les résultats enfin d'une aussi déplorable direction sur les affaires intérieures et sur les affaires extérieures de l'État ne peuvent manquer d'inspirer à l'ambassadeur de la République et un grand zèle pour démêler toutes les causes à cet état et une grande circonspection pour ne pas en augmenter le mouvement et en aggraver l'influence par une indiscrète et dangereuse intervention. » ${ }^{25}$

C'est peut-être à cause de tout cela que la situation politique espagnole n'inquiéta point Bonaparte et il ne pensa à l'Espagne que comme un simple auxiliaire matériel, sans rapport avec les affaires principales du moment. Il se limita à utiliser l'alliance pour des affaires secondaires, non sans intérêt pour lui, telles que les demandes d'un emprunt, d'aide navale pour les objectifs des Français à Malte et en Égypte (affaire qui serait longtemps un obstacle aux relations bilatérales à cause du refus permanent espagnol) ${ }^{26}$ et de servir d'intermédiaire auprès du Saint-Siège.

Bien que pour Talleyrand et pour la grande majorité des hommes politiques français l'alliance avec l'Espagne ne donnerait les fruits souhaités qu'une fois effectuée une réforme politique substantielle dans ce pays, personne ne doutait de l'intérêt de maintenir les meilleures relations et, donc, il fallait changer le style diplomatique dominant durant le Directoire. Dès le début, le Consulat assuma cette tâche, en commençant par un changement formel. On reprit immédiatement l'ancienne pratique, interrompue dès le commencement de la période révolutionnaire, d'échanger des cadeaux entre le roi et le Premier ministre ${ }^{27}$ et lorsqu'on envoya Alquier à l'ambassade, on lui ordonna d'être extrêmement prudent dans ses relations personnelles et de s'éloigner totalement des cercles politiques et des luttes internes. Dans ses rapports avec le gouvernement, Alquier devait maintenir une grande circonspection et dans ses rapports avec toutes sortes d'individus du royaume, «l'ambassadeur de la République n'a qu'à se resouvenir qu'il représente le 
gouvernement d'une Nation éminemment distinguée par l'urbanité de ses mœurs et la politesse de ses manières. ${ }^{28}$

Néanmoins, les faits démentirent immédiatement les mots. Le Consulat maintint, en apparence, une attitude qui n'était pas partisane envers la lutte politique interne espagnole et il traita de la même façon Urquijo et Godoy, mais Alquier ne tarda pas néanmoins à établir des relations intéressées ${ }^{29}$. D'autre part, le fait de traiter Urquijo et Godoy de la même manière constituait en soi une prise de parti, ou du moins on l'interpréta ainsi en Espagne. Godoy avait été durement attaqué par le Directoire et il dut abandonner le gouvernement en 1798 à cause de cela. Le changement d'attitude du Consulat envers lui le favorisa donc politiquement. Le premier signe évident dans ce sens se manifesta quelques jours après Brumaire. Le 22 novembre, Talleyrand, dans un rapport aux Consuls, rappelait que lors de la signature de la paix de Bâle, la France s'était engagée à offrir une armure à Godoy et ce n'était toujours pas fait. Malgré le coût de ce cadeau, 20000F environ, Bonaparte donna son accord et on l'envoya à Godoy. Quelques mois plus tard, en avril 1800, l'ambassadeur Alquier visita Godoy à son domicile, démarche inusitée puisque Guillemardet n'avait jamais fait une chose pareille. Le geste d'Alquier n'était pas gratuit, mais motivé par de fortes raisons qu'il exposa à Talleyrand et que celui-ci approuva: étant donné la situation politique espagnole, le partido de Godoy a de grandes possibilités face à celui d'Urquijo « et nous accueillerait mal, sans doute, si nous attendions le lendemain de la victoire pour nous en rapprocher. $»^{30}$

17 Les démonstrations officielles de considération envers Godoy n'empêchèrent pas Alquier de faire tout son possible pour le dénigrer. Dans ses célèbres et pittoresques dépêches diplomatiques, truffées d'inexactitudes, l'ambassadeur français transmit une image complètement négative du favori des souverains et se montra toujours, à titre personnel, favorable au maintien d'Urquijo au pouvoir, comme il le manifesta à Talleyrand le 18 août 1800, alors que le harcèlement envers Urquijo se renforçait en Espagne $^{31}$. Pourtant, Alquier ne put convaincre Bonaparte ni de l'intérêt du maintien au pouvoir d'Urquijo, ni de celui d'une intervention directe du Consulat dans les affaires internes espagnoles ${ }^{32}$ et quand celui-ci envoya son frère Lucien comme ambassadeur en Espagne, le Premier Consul se déclara en faveur de Godoy comme homme fort de la politique au sud des Pyrénées. En effet, Lucien devint immédiatement ami du favori, et cela lui permit de lui rendre visite à son domicile sans le prévenir, chose extraordinaire qui surprit la Cour espagnole ${ }^{33}$. L'amitié entre Lucien Bonaparte et Godoy prouva que le Premier Consul avait pris parti. Il n'est donc pas surprenant que le 13 décembre, seulement dix jours après l'arrivée de Lucien à l'Escorial, se produisit la destitution d'Urquijo comme secrétaire d'État. Le changement en Espagne, après trois ans d'une intense lutte partisane, se matérialisait.

La coïncidence de dates n'est pas fortuite, mais toutefois il ne faudrait pas attribuer uniquement au Consulat le changement politique espagnol. La lutte pour le pouvoir était motivée en Espagne par de strictes raisons internes, et Alquier le comprit ainsi quand, en août 1800, il informa Talleyrand de la possibilité d'un prochain changement de gouvernement en Espagne, causé exclusivement par les erreurs de la reine et la croissante ambition de Godoy ${ }^{34}$. Pour en finir avec Urquijo, l'intervention française n'était pas nécessaire, étant donné la quantité d'ennemis qu'il avait à l'intérieur du pays : la reine et Godoy ${ }^{35}$ ne le supportaient pas pour des raisons personnelles; l'Inquisition, la hiérarchie ecclésiastique et le nonce apostolique, Monseigneur Casoni, 
s'opposaient à lui à cause de sa politique religieuse ; son accord en politique extérieure avec la France, position clairement alignée sur celle de Godoy lors de l'étape antérieure, suscita l'opposition des aristocrates du partido inglés. Tout cela motiva, en 1799-1800, la multiplication des machinations et des intrigues contre lui; on l'accusait même de détournement de fonds et d'enrichissement personnel illicite, à tel point que le roi luimême y crut ${ }^{36}$. Cependant, Urquijo put se maintenir au pouvoir plus longtemps que prévu et sa révocation n'arriva qu'à la fin de 1800 , coïncidant avec l'affirmation du nouveau régime en France et l'arrivée en Espagne de Lucien comme ambassadeur. Trop d'indices pour ne pas penser que même si les raisons de la chute d'Urquijo sont de caractère interne, sa position politique s'affaiblit réellement après le changement politique effectué en France.

19 Certes, le tournant politique de la France après Brumaire fut néfaste pour Urquijo pour deux raisons fondamentales: le rapprochement de Bonaparte avec le Saint-Siège et l'opinion négative du Premier Consul sur le ministre espagnol. L'autorisation officielle, le 28 décembre 1799, du culte catholique en France fut interprétée en Espagne comme un changement radical du nouveau régime envers l'Église catholique, impression confirmée par l'ouverture de pourparlers en vue de la signature du Concordat, à partir de l'élection du nouveau pape, Pie VII en mars de l'année suivante. Tout cela confirma que la politique d'opposition d'Urquijo envers le Saint-Siège n'était pas indiquée et Charles IV, dès qu'il connut l'élection du nouveau pape, s'empressa d'apporter le témoignage de son adhésion à Rome et de son changement de politique : il nomma un ambassadeur au Saint-Siège, abolit rapidement le décret de septembre de l'année précédente, véritable étendard d'Urquijo, et changea radicalement son attitude auprès du Saint-Siège. Au lieu d'intervenir en matière ecclésiastique, il décida de négocier à l'amiable toute sorte d'affaires, même les plus épineuses ${ }^{37}$. Après Brumaire, il existait en Espagne la conviction que la politique de force, plus ou moins stricte, suivie à l'époque du Directoire à l'égard du Saint-Siège, n'était pas la plus indiquée. Le nonce apostolique Casoni se rendit également compte du changement et redoubla ses attaques contre Urquijo. Le fait définitif, néanmoins, fut l'intervention personnelle de Pie VII qui envoya à Charles IV une lettre interprétée, à juste raison, comme une prise de position contre le Premier ministre espagnol. Le pape conseillait au roi : «que cerrase sus oídos a los que, so color de defender las regalías de la Corona, no aspiraban sino a excitar aquel espíritu de independencia que, empezando por resistir al blando yugo de la Iglesia, acaba después por hacer beberse todo el freno de obediencia y sujeción a los gobiernos temporales» ${ }^{38}$. Dans la nouvelle conjoncture internationale, et quand on avait en Espagne l'image d'un Bonaparte disposé à en terminer avec la Révolution en France et à régler les différends avec le Saint-Siège, cet appel à l'ordre du pape était plus que déterminant pour Charles IV et aurait dû être suffisant pour destituer Urquijo.

Mais de plus, Urquijo avait aussi à souffrir de l'opposition de Bonaparte. Sur ce point l'attitude d'Azara, surtout lors de la conversation avec le général français le 17 octobre, donc antérieure à Brumaire, fut décisive. Dans ses mémoires, Azara lui-même décrit comment, pendant plus de trois heures, ils parlèrent de la situation politique en France et des «motivos de mi remoción [comme ambassadeur à Paris] y el estado en que se hallaban los negocios en mi Corte" ${ }^{39}$. À cette époque Azara se montrait particulièrement critique envers Urquijo, qu'il rendait responsable de ses problèmes personnels et de toutes les difficultés politiques de l'Espagne ${ }^{40}$. Son opinion fut sûrement prise en compte par Bonaparte. Sans doute cet ambassadeur espagnol destitué dut raconter en détail les dangereuses amitiés d'Urquijo et sa relation, plus que 
circonstancielle, avec les jacobins le 30 prairial. Selon Azara ${ }^{41}$, Urquijo avait comme principal confident à Paris le consul espagnol José de Lugo, chez qui se réunissaient « los más encarnizados terroristas enemigos de toda Monarquía »- («les terroristes les plus acharnés de toute la monarchie») - parmi lesquels il cite plusieurs Espagnols (Gimbernat, Marchena, l'épouse de Gonzalo O'Farril) et quelques Français, dont il ne révèle pas les noms, sauf celui de l'ancien conventionnel Paganel, agent de liaison entre Urquijo et le jacobinisme français. D'autre part, on connaissait l'amitié entre Urquijo et Valkenaer, le représentant batave en Espagne, dont les opinions jacobines - et activités économiques peu transparentes - étaient très connues. Bonaparte ne resta pas indifférent à cette information et il exigea de l'Espagne l'immédiate destitution de Lugo. Le 7 décembre 1799, Talleyrand communiquait à l'ambassadeur à Paris que le Consulat refuserait l'exequatur à Lugo pour la prolongation de ses fonctions comme consul général à Paris et, malgré la résistance d'Urquijo, Lugo abandonna Paris ${ }^{42}$.

21 Bonaparte avait d'autres raisons personnelles contre Urquijo. Celui-ci avait refusé avant Brumaire la traduction en espagnol de deux textes sur les campagnes d'Italie et d'Égypte et durant les premiers mois du Consulat, il avait fait une confidence fâcheuse à Alquier sur le manque d'autorité du Premier Consul et sur sa rivalité avec son frère Lucien ${ }^{43}$, opinion connue très vite par les représentants diplomatiques à Madrid, comme l'informa l'ambassadeur danois, Schubart, dans une lettre envoyée à sa sœur en 1800 : «Valkenaer et Urquijo ne croient pas à la stabilité du nouveau Gouvernement françois» et n'apprécient pas non plus le remplacement de Guillemardet, "car ils avoient su le gagner par des intrigues et des largesses. ${ }^{44}$

Les ressentiments personnels et la relation d'Urquijo avec le jacobinisme influencèrent Bonaparte beaucoup plus que d'autres conflits avec l'Espagne, si fréquents au cours des premiers mois du Consulat, en particulier à cause des problèmes suscités à la suite du refus espagnol à la demande réitérée d'aide navale. Urquijo lui-même s'en rendit compte et se considéra obligé de se justifier personnellement auprès de Bonaparte. Le 12 janvier 1800, il envoya à l'amiral Mazarredo ${ }^{45}$ une lettre en français, pour en faciliter la lecture au Premier Consul, dans laquelle il essayait de démentir, tout d'abord, les informations à son sujet reçues par le citoyen Bonaparte : «ce magistrat dans le premier moment de ses fonctions importantes n'a pu fixer son opinion au sujet du gouvernement espagnol d'après un examen bien approfondi et fait par lui-même, mais d'après une connaissance imparfaite des hommes et des choses qui ont influé dans les relations qui existent entre les deux Puissances alliées ». Il est évident qu'Urquijo supposait qu'Azara avait été la principale source d'information de Bonaparte, et c'était pour cela qu'il essayait de justifier sa destitution en tant qu'ambassadeur, invoquant des raisons telles que l'ambassadeur «n'avait su se maintenir aussi étranger aux factions qu'on ne le désirait ici » et en réaffirmant que son animosité n'y était pour rien. C'est-à-dire qu'Urquijo accusa Azara pour sa participation aux événements de prairial en faveur des réalistes, tandis qu'Azara l'accusait exactement du contraire. Il finissait sa lettre en louant Brumaire, «la dernière révolution», de laquelle il attend la consolidation du pouvoir en France, car les factions politiques à l'intérieur disparaitront et le processus de paix s'accélèrera et il promet l'envoi de navires et de vivres demandés par Bonaparte pour aider l'armée en Égypte, en signe de considération envers le Premier Consul ${ }^{46}$. L'effort fut important, mais vain. Bonaparte opta finalement pour Godoy et traita avec celui-ci les affaires fondamentales de politique extérieure, une fois venu le moment de compter sur l'Espagne. 
L'engagement extérieur de l'Espagne

Quelques mois après l'instauration du Consulat, la Cour espagnole était convaincue de la capacité de Bonaparte à mettre de l'ordre à l'intérieur de la France et à dominer en Italie, et on crut à l'opportunité d'agrandir le duché de Parme, objectif principal de Charles IV en politique extérieure. Le gouvernement fit confiance donc à la nouvelle conjoncture politique française et à la bonne disposition envers l'Espagne exprimée à la fin mars 1800 par le Premier Consul et prétendit entamer des pourparlers dans ce but ${ }^{47}$. Il s'agissait d'une initiative prématurée car, à ce moment-là, la France n'exerçait aucun contrôle effectif sur les territoires italiens, tandis qu'au contraire l'Autriche continuait à y maintenir son influence, qui était l'obstacle fondamental aux projets espagnols. Bonaparte ne montrait, lui non plus, aucun intérêt à l'accomplissement de ces projets, mécontent en plus de la réticence espagnole à lui accorder l'aide navale nécessaire pour secourir Malte et l'armée française d'Égypte ${ }^{48}$. Tout semblait donc indiquer que, malgré les illusions espagnoles, le changement de régime en France n'allait impliquer sur ce point aucune innovation importante par rapport à la politique du Directoire. Néanmoins, à partir du moment où Bonaparte, victorieux à Marengo, laissa hors de cause l'Autriche, après la convention d'Alexandrie, il accepta de traiter l'extension du territoire de Parme. Le 22 juillet 1800, le Premier Consul envoya des pouvoirs à Alquier pour ouvrir des pourparlers avec le gouvernement espagnol et quelques mois plus tard, le 1er octobre, on signait à San Ildefonso de la Granja, la résidence d'été des monarques, le Tratado preliminar y secreto entre España y la República francesa sobre el engrandecimiento del ducado de Parma y la retrocesión de Luisiana, négocié par Urquijo et le général Berthier, envoyé spécial du Premier Consul ${ }^{49}$. Il ne s'était pas passé plus d'un an depuis Brumaire et, apparemment, tout indiquait que le nouveau régime français donnerait les fruits attendus par l'Espagne. Cependant, la réalité fut bien différente et on put observer immédiatement que les relations avec Bonaparte obligeaient la monarchie espagnole à pratiquer une politique extérieure d'engagement extrême et de risque excessif pour ses possibilités matérielles et sa capacité militaire.

Dans l'accord sur Parme, la France s'engageait à agrandir les États du duché avec les territoires de la Toscane ou des Légations romaines (on opta finalement pour les premiers), jusqu'à obtenir une population de 1200000 habitants. L'Espagne céderait la Louisiane et mettrait à disposition de la France six navires de guerre avec 74 canons, prêts à recevoir les équipements et le ravitaillement français. Urquijo, satisfait de l'accord puisqu'il accomplissait une aspiration historique des souverains espagnols, s'efforça de démontrer que la Louisiane coûtait à l'Espagne plus qu'elle ne le valait et qu'il était donc avantageux de l'échanger contre la Toscane, le royaume où avait fleuri la Renaissance, comme disait Godoy, avec une certaine ironie, dans ses Mémoires. L'accord surprit la Cour espagnole qui l'interpréta comme un acte de " generosidad del dueño de Europa » - («de générosité du maître de l'Europe») - et l'attribua au climat d'amitié créé à partir de Brumaire et qui contrastait avec le comportement du Directoire ${ }^{50}$. On croyait que le Consulat inaugurait un temps propice aux intérêts extérieurs espagnols. Ce ne fut qu'une illusion. Avec la possession de la Toscane, très tôt appelée royaume d'Étrurie à cause de la fascination de Bonaparte pour l'Antiquité, l'Espagne devenait son otage en Italie et perdait son autonomie ${ }^{51}$. À partir de ce moment-là, l'influence, plus ou moins réelle, reconnue aux Bourbons espagnols dans certaines régions italiennes disparaissait et la dépendance complète vis-à-vis de Bonaparte se faisait évidente. Pour couronner cela, le Premier Consul essaya de 
retarder et de compliquer la prise de possession du royaume d'Étrurie par son nouveau titulaire Louis de Bourbon, héritier du duc de Parme et époux d'une fille de Charles IV, et les soumit tous deux à l'humiliation (extensible à la Cour espagnole, bien que celle-ci simulât l'ignorance) de les obliger à passer par Paris avant de s'installer dans leur royaume.

L'affaire de Parme fut toujours soumise à la politique de Bonaparte vis-à-vis de l'Autriche et, en dépit de l'interprétation espagnole, les désirs de Charles IV et de son épouse ne comptèrent guère. Bonaparte fit à cette occasion une bonne affaire, en utilisant son allié : avec la Louisiane, il récupéra pour la France un territoire très utile pour une possible négociation avec l'Angleterre, il laissa sous la responsabilité de l'Espagne le contrôle stratégique du port de Livourne, disputé par les Britanniques et les Napolitains, il obtint des arguments pour accroître la demande d'aide navale de l'Espagne et il démontra sa capacité pour créer des royaumes en Italie. D'autre part, l'enthousiasme espagnol pour le résultat permit au Premier Consul d'augmenter les exigences présentées à son allié. Quand il fut sur le point de signer l'accord sur Parme, Bonaparte exposa son désir que l'Espagne puisse conquérir le Portugal ${ }^{52}$ en très peu de temps et, un mois après, il envoya son frère Lucien comme ambassadeur à Madrid, avec la mission de concrétiser ce compromis, qu'il devait négocier non pas avec le Premier ministre, Urquijo, mais avec Godoy, le seul homme capable, selon l'opinion générale de l'époque, de contrecarrer les réticences de Charles IV à entreprendre une guerre avec le pays où sa fille aînée était l'épouse du chef de l'État, le régent don Joao. Ainsi donc, lorsque fin 1800, Bonaparte se proposa comme objectif principal de harceler l'Angleterre à partir du Portugal, il n'hésita pas à avoir recours à celui qu'il estimait le plus apte pour cette tâche, indépendamment du fait que Godoy fût légalement ou pas le responsable de la politique extérieure espagnole.

Bonaparte calcula, avec justesse, que Godoy ne s'opposerait pas à ses exigences, car en pleine dispute pour le pouvoir au sein de la monarchie espagnole, il aurait besoin de l'appui du «maître de l'Europe ». Il savait de même qu'il pouvait compter sur l'accord de la reine, la personne la plus intéressée à installer sa fille sur le trône de Toscane. L'engagement de l'Espagne au Portugal était ainsi assuré et, par conséquent, sa collaboration au programme de politique extérieure du Premier Consul. À cette occasion, l'Espagne serait complètement intégrée dans ses objectifs internationaux, bien que, une fois de plus, Bonaparte se réservât l'initiative et le choix du temps. Le 22 décembre 1800 , il ordonna à son frère d'insister pour la déclaration de guerre de l'Espagne au Portugal ${ }^{53}$ et le 29 janvier 1801 Lucien et Godoy signèrent un accord à Madrid, par lequel ils décidèrent l'envoi d'un ultimatum au Portugal si celui-ci ne rompait pas complètement avec l'Angleterre. Un mois après, le 27 février, l'Espagne déclara la guerre qui fut retardée pendant un temps, précisément à cause des hésitations de Bonaparte lui-même dues à certains changements conjoncturels dans le panorama international européen. Mais lorsqu'il estima opportune l'invasion du Portugal par les troupes espagnoles, la célèbre Guerra de las Naranjas se produisit. L'engagement pris alors par l'Espagne pèserait les années suivantes de façon extraordinaire et conditionnerait sa politique intérieure et extérieure jusqu'à la rupture définitive de 1808 avec l'Empire français.

27 En dépit de ce qui était stipulé dans le traité d'alliance de 1796, depuis Brumaire Bonaparte agit toujours sans se soucier des intérêts diplomatiques espagnols. Son insistance à disposer de navires espagnols pour secourir les troupes françaises en 
Égypte durant les premiers mois de son gouvernement provoqua de grosses difficultés pour l'Espagne, car la Turquie fut sur le point de rompre ses relations diplomatiques avec elle et il existait le risque, comme Urquijo le signala à plusieurs reprises, d'étendre le conflit aux États nord-africains qui suivraient son exemple, ce qui aurait placé l'Espagne dans une situation compliquée car le commerce du blé avec ces pays-là était d'une importance capitale ${ }^{54}$. D'autre part, Bonaparte fit toujours abstraction des considérations habituelles et formelles avec un allié. D'abord, Urquijo et ensuite son successeur dans la diplomatie espagnole, Pedro Ceballos, et même Godoy, l'authentique homme fort de la monarchie, exposèrent continuellement leurs plaintes pour le manque d'informations sur les conversations et accords de Bonaparte avec d'autres pays, même lorsque les intérêts espagnols entraient en jeu ${ }^{55}$. De plus, Bonaparte n'hésita pas, lorsque cela l'intéressait, à éluder l'accomplissement de certains accords avec l'Espagne, qu'il avait lui-même promus. Il en fut ainsi avec la convention maritime signée par les deux pays le 13 février 1801, selon les ordres transmis par Bonaparte à son frère Lucien, dont les plans ne purent jamais être exécutés, le Premier Consul les ayant estimés inutiles peu de temps après la signature ${ }^{56}$. Bref, la monarchie espagnole vécut ses relations avec Bonaparte sans jamais savoir, ni à court ni à long terme, ce qu'il pourrait advenir des accords et des traités signés. La situation fut parfaitement décrite en 1801 par Azara dans l'un de ses rapports adressés au secrétaire d'État espagnol, Pedro Ceballos : « No se admire V.E de la rapidez con que aquí se varían los planes y las convenciones, porque ya las gentes se han acostumbrado a semejantes contradicciones y a ver por la tarde deshecho quanto se ordenó por la mañana. Esto en gran parte proviene del carácter y disposición del Primer Cónsul. Parta V.E del principio cierto de que aquí no se creen obligados a mantener ningún convenio ni tratado. " ${ }^{57}$

La nouvelle politique de Bonaparte se heurta aux usages de la Cour espagnole. Ce que cette dernière interpréta comme le début d'une époque propice, devint une succession de problèmes qui engagèrent l'Espagne dans une situation internationale complexe, absolument défavorable pour ses intérêts. D'autre part, les gouvernants espagnols ne comprirent en aucun cas le caractère du personnage qui, selon eux, devait être le restaurateur de la monarchie en France aux lendemains de Brumaire. Comme le perspicace Azara l'écrivait à son ami Iriarte, «Bonaparte se cree muy superior a cuanto hay y ha habido en el mundo y dile al Príncipe de la Paz que me crea si le advierto que no tiene ideas justas de lo que es este hombre, ni de sus travesuras " ${ }^{58}$. Ce qui est démontré, c'est qu'au début du Consulat, la monarchie espagnole fit trop confiance au nouveau régime français et fut incapable de s'adapter ou de s'opposer aux nouveaux usages introduits, tout d'abord par le général Bonaparte et peu après par l'Empereur Napoléon.

\section{NOTES}

[2月. Cette étude a été réalisée dans le cadre du projet de recherche n PB97-0200 (Programa Sectorial de Promoción General del Conocimiento. Ministerio de Educación y Cultura. Espagne). 
1.F. Saavedra remplaça Manuel Godoy au secrétariat d'État au mois de mars 1798. Lorsqu'il tomba malade quelques mois plus tard, il fut remplacé par Urquijo. Urquijo fut l'acteur principal de la politique espagnole entre 1798 et 1800.

2.Le 5 septembre 1799, au moment où, après la mort de Pie VI, il y avait une vacance du siège papal, Urquijo publia un décret par lequel les évêques espagnols obtenaient le droit, réservé jusque-là au souverain pontife, d'accorder des dispenses dans certains cas d'empêchements matrimoniaux. Même si ce droit était déjà reconnu dans d'autres pays, la mesure restait surprenante en Espagne et elle devint une espèce de symbole de la politique de réformes du gouvernement d'Urquijo. Le décret suscita de sérieuses controverses parmi les ecclésiastiques, mais ce fut surtout sa signification politique qui provoqua un sérieux affrontement entre les partidos qui se disputaient le pouvoir politique en Espagne.

3.Selon Schubart, l'ambassadeur danois à Madrid, Urquijo connaissait bien l'importance des forces de l'opposition, mais il espérait se maintenir au pouvoir grâce à l'orientation politique du Directoire. (Archives du Ministère des Affaires Étrangères, Paris, Correspondance Politique, Espagne, supplément, $\mathrm{n}^{\circ} 26, \mathrm{pp}$. 303-304.)

4.Le Directoire participa directement au renversement de Godoy en mars 1798 et, un an plus tard, le gouvernement français tenta de provoquer la révocation d'Urquijo, en faisant pression directement sur Charles IV. Cf.Emilio LA PARRA, La alianza de Godoy con los revolucionarios (España y Francia a fines del siglo XVIII), Madrid, CSIC, 1992 et, du même auteur : « La crisis política de 1799 » in Revista de Historia Moderna, nos 8-9 (Alicante, 1990), pp.219-232.

5. « Le nouveau régime modifiera totalement la Constitution de la France et la politique de toute l'Europe. »

6.Les paroles de l'ambassadeur reflétaient son évaluation positive : « ...todo el buen partido de la Francia está a favor de la innovación porque se promete con mucho fundamento la paz general y la tranquilidad interior del Estado», dépêche du 11 novembre 1799, (Archivo Histórico Nacional, Madrid, Estado, 3990).

7.Le 18 novembre, l'ambassadeur affirme que les nominations politiques ont touché des " personas muy distinguidas por sus talentos y probidad, de manera que merecen la aprobación general »- («des personnes distinguées par leurs talents et leur probité qui méritent ainsi l'approbation générale») - et, le 5décembre, il fait l'éloge de la nomination d'Alquier à l'ambassade de Madrid, en le présentant comme une personne «muy a propósito » pour ce poste (AHN, Estado, 6686).

8.Ce renforcement de l'Exécutif « produira les meilleurs effets et pourra contenir les insurrections de l'intérieur. »

9. "Grâce aux réformes salutaires qui se mettent en place dans toutes les branches de l'administration et qui produisent le meilleur effet, le gouvernement acquiert ainsi chaque jour une faveur méritée auprès de l'opinion ». Dépêches du 17 décembre 1799 et du 11 janvier 1800 (AHN, Estado, 3963). Il convient de préciser que Guillemardet, l'ambassadeur français, dans un communiqué officiel à la Cour d'Espagne au sujet de Brumaire, avait signalé les mêmes réussites du nouveau régime que Múzquiz avait communiquées : la recherche de la paix, l'ordre dans l'administration, le rétablissement de la tranquillité intérieure et la rédaction d'une constitution (Guillemardet à Urquijo, 29 novembre 1799, in AHN, Estado, 6686).

10.« On ne parle pas pour le moment de lui donner un commandement dans l'armée, mais il est normal qu'il souhaite désormais tirer parti de l'ascendant qu'il a sur les autres généraux et sur les Français » (AHN, Estado, 6689). 
11.Múzquiz fut une des rares personnes qui, quand il était ambassadeur à Berlin, son affectation antérieure, eut des relations assidues avec Sieyès, son homologue français. Ils étaient tous les deux taciturnes et ils avaient un mode de vie très paisible (Andrés MURIEL, Historia de Carlos IV, Madrid, B.A.E., 1959, II, p. 122).

12.« Vous pouvez être sûr qu'avant ventôse, la moitié de la France se donne au diable avec cette révolution des baïonnettes. »

13.«Mon ami Perico : je suis d'accord avec vous concernant ce pays et votre lettre du 18 m'a beaucoup plu ». Les deux lettres à l'AHN, Estado, 3990. Gómez Labrador se basait sur l'impopularité du remplacement de l'emprunt forcé, abrogé par le nouveau régime, par un autre de cinq livres sur la contribution foncière, personnelle, mobilière et somptuaire et la prévisible approbation de nouveaux impôts sur le sel et autres denrées.

14.André Fugier, Napoléon et l'Espagne, Paris, Alcan, 1930, I, p. 88, remarque qu'Urquijo reçut cette nouvelle avec plaisir, mais il ne donne pas la source de cette affirmation.

15.« Je n'ai des égards pour ces gens qu'en raison de nos affaires ; je répète que je ne les estime pas, je ne les aime pas, ni ne les craint, et que je souhaite seulement les abandonner dès que je le pourrai.»

16.« Il est encore presque révolutionnaire, il ne manque chez ses membres ni orgueil, ni violence, mais de l'instabilité et de la vanité.»

17.« où je n'ai que des désagréments " (Múzquiz à Urquijo, 9 février 1800, AHN, Estado, 3963).

18." La pacification des chouans continue à faire des progrès rapides... et on ne doute pas que l'on verra bientôt une heureuse conclusion à une si funeste guerre » (AHN, Estado, 3963).

19.« À cause de l'hostilité ouverte dont faisait preuve le Premier Consul à l'égard des idées et des passions démagogiques.»

20.Godoy signale (PRINCIPE DE LA PAZ, Memorias, Madrid, B.A.E., 1965, I, p. 292) que Charles IV craignait quelque peu que la France, dirigée par Bonaparte, augmentât son pouvoir et modifiât ainsi la situation internationale, c'est-à-dire que la dépendance de l'Espagne en fût accrue. Mais il se pourrait qu'il s'agisse davantage d'une remarque personnelle de Godoy que l'expression de la pensée du roi à cette époque, enthousiasmé - semble-til - par les perspectives internationales qu'offrait Bonaparte en Italie. Voir également Andrés MURIEL, op. cit., II, p. 182.

21. « La grande République démocratique et philosophique de la France est tombée, le gouvernement réellement populaire et démocratique n'existe plus, remplacé par un gouvernement aristocratique et quasi monarchique » (Diario de Luengo, cité par Rafael OLAECHEA, El cardenal Lorenzana en Italia, León, 1981, pp.280-281.

22.Lettre de Lucien au Premier Consul du 24 février 1801, citée par François PIETRI, Luciano Bonaparte (El hombre que no quiso ser rey), Barcelone, Juventud, 1942, p. 136. 23. "Bonaparte n'a pas d'ami plus franc et loyal que Charles IV. Il sait aussi bien gouverner que se battre... c'est un grand homme que j'aime de tout mon cœur, un aussi bon catholique que moi ! (Dépêche d'Alquier du 12 novembre 1800, citée par FUGIER, op. cit., I, p. 111). L'allusion à la religiosité de Bonaparte était le meilleur éloge pour Charles IV. Comme Alquier l'avait déjà écrit, « le moyen le plus sûr d'exciter ses préventions, c'est de lui dire de quelqu'un qu'il n'a pas de religion», dépêche du 18octobre 1800, in Henri PERRIN DE BOUSSAC, Charles-Jean-Marie Alquier (1752-1826), La Rochelle, 1983, p.114. 
24.André FUGIER, op. cit., I, p. 92.

25.AAE, Corresp. pol. Espagne, 659 (cité par H. PERRIN DE BOUSSAC, op. cit., p. 111).

26.L'Espagne octroya, sans difficulté, un prêt d'un million et demi de piastres pour ravitailler la flotte de l'amiral Villaret-Joyeuse affectée à La Plata, mais avec un intérêt élevé (cf. François PIETRI, Lucien Bonaparte à Madrid (1801), Paris, Grasset, p. 70). Le soutien naval posa plus de problèmes, entre autres raisons parce que la flotte espagnole, depuis des années, manquait singulièrement de moyens en hommes et en matériel pour armer les navires exigés par Bonaparte (voir le rapport du ministre de la Marine, Juan de Lángara, AHN, Estado, 6686).

27.André FUGIER, op. cit., I, pp. 97-98.

28.Instructions à Alquier (AAE, Corresp. pol. Espagne, 659 (cit. par Henri PERRIN DE BOUSSAC, op. cit., p. 111).

29.Alquier ne manqua pas de se mettre en rapport avec différentes personnalités compromises dans des intrigues politiques, ce qui n'a guère plu à la reine et à Godoy. Les marques de confiance et les visites répétées, au printemps 1800, d'Alquier au duc d'Osuna, l'un des chefs de l'opposition aristocratique à Godoy, préoccupèrent la reine qui fit part à Godoy de ses craintes concernant le soutien français à ce groupe (Lettres de la reine à Godoy du 8 et du 12 mars 1800, in Carlos PEREYRA, Cartas confidenciales de la reina Ma Luisa y de don Manuel Godoy, Madrid, Aguilar, s/d, pp. 268-269 et 270).

30.Dépêche d'Alquier à Talleyrand, citée par Henri PERRIN DE BOUSSAC, op. cit., p. 121, cf. André FUGIER, op. cit., I, pp. 120-121.

31.Dépêche du 18 août 1800, citée par Luciano TAXONERA, Godoy, príncipe de la Paz y de Bassano, Barcelone, Juventud, 1946, p. 169.

32.Le 20 mai 1800 (ANP, AFIV 1679), Alquier demanda l'autorisation du Premier Consul pour tirer parti de la multiplicité des « fantaisies » des souverains espagnols. Il semble que Bonaparte ne donna pas son consentement.

33.Dès le début de 1800 , c'est-à-dire alors même que le Consulat se consolidait comme régime politique, le crédit d'Urquijo était presque nul aux yeux des puissances européennes. La France préférait négocier avec Godoy les affaires les plus importantes, de même que l'Angleterre, dont le gouvernement donna des instructions pour engager une négociation secrète le 1er février 1800. (André FUGIER, op. cit., I, p. 118, n 1 ).

34.Dépêche d'Alquier du 8 août 1800, cit. par Luciano TAXONERA, op. cit., pp. 162-166. 35.Les mémoires des contemporains et les dépêches des diplomates étrangers sont d'accord sur la suffisance d'Urquijo et l'air de supériorité avec lequel il traitait presque tout le monde à la Cour, même la reine et Godoy.

36. Marqués de LEMA, Antecedentes políticos y diplomáticos de los sucesos de 1808, Madrid, 1912, p.21.

37.Voir l'exposé de ce changement politique in R. OLAECHEA, op. cit., p. 302.

38. "Qu'il n'écoute pas ceux qui, sous prétexte de défendre les droits de la Couronne, n'aspirent qu'à attiser un esprit d'indépendance qui, commençant par résister au faible joug de l'Église, s'achève ensuite par un refus de toute obéissance et sujétion aux gouvernements temporels » (cité par PRÍNCIPE DE LA PAZ, Memorias, I, pp. 308-309).

39.« Des motifs de mon remplacement [comme ambassadeur à Paris] et de l'état dans lequel se trouvaient les affaires à la Cour » (José Nicolás de AZARA, Memorias, éd. de Gabriel Sánchez Espinosa, Frankfurt am Main, Peter Lang, 1994, p. 485).

40.José GARCía DE LEÓN PIZARRo, Memorias, Madrid, Instituto de Estudios Políticos y Constitucionales, 1998, p. 94. Il affirme qu'à cette époque Azara agissait en ennemi 
déclaré, et même en ennemi « personnel » d'Urquijo et qu'il ne ratait pas une occasion de l'attaquer.

41.Azara à Godoy, 26 novembre 1799, cité par Gabriel Sánchez Espinoza, in José Nicolás de AZARA, op. cit., p. 523.

42.AHN, Estado, 3990.

43.André FUGIER, op. cit., I, p. 95.

44.Herman de SCHUBART, «Lettres d'un diplomate danois en Espagne», Revue Hispanique, 1902, p. 426.

45.L'ambassadeur d'Espagne à Paris était alors Múzquiz, mais Urquijo accorda à Mazarredo, commandant de la flotte espagnole mouillée à Brest, de larges pouvoirs diplomatiques au point qu'il agissait comme un autre ambassadeur.

46.AHN, Estado, 3963.

47.André FUGIER, op. cit., I, pp. 107-108 Jack BERTE-LANGEREAU, La política italiana de España bajo el reinado de Carlos IV, Madrid, Revista de Occidente, 1958, p. 95.

48.Dès la fin du Directoire, la tension diplomatique entre la France et l'Espagne s'accrut à cause de la demande française d'aide navale à l'Espagne. Bonaparte intensifia cette demande, car il prétendait, dès le début, disposer de la flotte espagnole mouillée à Brest selon son bon vouloir, et disposer plus largement des navires espagnols pour venir au secours de Malte et de l'armée d'Égypte. Le refus répété de l'Espagne d'accorder cette aide, de par l'impossibilité matérielle et la différence entre les stratégies des deux pays (l'Espagne souhaitait envoyer sa flotte pour libérer Trinidad et Mahón, aux mains des Britanniques), provoqua un échange continuel de plaintes (in AHN, Estado, 5205, on trouve de nombreuses dépêches diplomatiques à ce sujet. Voir également AAE, Corresp. pol. Espange, 657, p. 290).

49.Pour le texte du traité en français, voir AN, AFIV, 1610, plaq. 1.

50.PRínCIPE DE LA PAZ, Memorias, I, p. 292.

51.André FUGIER, op. cit., I, p. 111.

52.Le 30 septembre 1800, Bonaparte écrit de manière catégorique : « Il est nécessaire que les troupes espagnoles soient maitresses du Portugal avant le 15 octobre » (cit. par André FUGIER, op. cit., I, p.136).

53.Marqués de LEMA, op. cit., p. 120.

54.Dépêche d'Urquijo à Múzquiz (AAE, Corresp. pol. Espagne, 657, pp. 287-288). Voir également les fréquentes dépêches à ce sujet entre les chargés d'affaires espagnols à Constantinople (d'abord Bouligny puis, à partir de 1800, Corral) et Azara, ainsi qu'avec le gouvernement espagnol, in AHN, Estado, 4768.

55.Le gouvernement espagnol fut informé des conversations du Consulat avec l'Autriche sur la situation en Italie et les préliminaires de Lunéville par son ambassadeur à Berlin. Le 2 janvier 1801, Cevallos, désespéré par l'attitude de Bonaparte, ordonnait à Múzquiz d'exposer les plaintes espagnoles (AHN, Estado, 5205). 56.Azara le révéla dans sa dépêche du 27 mars 1801 : « ... Puedo decir que el Cónsul no gusta que se diga ser nulo ni absurdo el tratado de operaciones marítimas que firmó su hermano con el Sr. Príncipe de la Paz, no obstante que está persuadido de ello. » (... je peux dire que le Consul n'apprécie pas que l'on dise que le traité des opérations maritimes signé par son frère, le Prince de la Paix, est nul et absurde, même s'il en est persuadé), (AHN, Estado, 5205).

57.«Que Votre Excellence ne s'étonne pas de la rapidité avec laquelle les plans et les conventions sont modifiés, car les gens se sont habitués à de telles contradictions et à revenir l'après-midi sur ce qui a été ordonné le matin. Ceci vient en grande partie du 
caractère et du tempérament du Premier Consul. Que Votre Excellence parte du principe suivant lequel on ne se croit pas obligé ici de conserver quelque convention ou traité que ce soit » (Dépêche du 30 avril 1801, AHN, Estado, 5205).

58. «Bonaparte se croit très supérieur à tout ce qui existe et qui a existé au monde, et dites au Prince de la Paix qu'il me croie si je le préviens qu'il n'a pas d'idées justes de ce qu'est cet homme, ni de ses ruses » (Azara à Iriarte, 20 décembre 1801, cit. par R. OLAECHEA, op. cit., p.282).

\section{RÉSUMÉS}

L'événement de Brumaire fut bien accueilli dans les milieux officiels espagnols. La Cour de Charles IV attendait du nouveau régime français l'aide nécessaire pour accomplir ses projets en politique extérieure, mais aussi quelque chose de plus important: la fin de la Révolution en France et, en conséquence, la fin des tentatives pour l'exporter en Espagne. Cette façon d'interpréter le revirement politique de la France créa un nouveau climat contraire au gouvernement dirigé par Mariano L. Urquijo, engagé dans une ambitieuse politique réformiste, et en décembre 1800 il fut obligé de démissionner de sa charge. Contrairement à ce qui arriva à l'époque du Directoire, il n'y eut aucune intervention directe française dans les affaires intérieures espagnoles, mais en politique extérieure l'influence de Bonaparte fut directe et déterminante et créa de nouvelles incertitudes sur la position internationale de l'Espagne, rendant plus difficiles ses relations avec la France et accroissant son engagement international. La monarchie espagnole fit trop confiance au nouveau régime de la France et fut incapable de s'adapter ou de s'opposer aux nouveaux usages introduits par le général Bonaparte.

Political Changes in Spain after Brumaire. The Brumaire coup was welcomed in official Spanish quarters. The court of Charles IV was expecting both support from the new French regime in order to attain its foreign policy goals and, more importantly, an end to the Revolution in France and hence a cessation of attempts to export it to Spain. This interpretation of the political change in France bred a climate that undermined the government led by Mariano L. de Urquijo, who was engaged in ambitious reformist policies, and resulted in his resignation in December 1800. Contrary to events during the Directory, there was initially no direct French intervention in Spanish domestic affairs, but in foreign policy Bonaparte's influence had a major impact, creating new uncertainties as to the international position of Spain, increasing her involvement abroad and complicating her relations with France. The Spanish monarchy had too much confidence in the new French regime and was unable to accommodate, let alone oppose the new agenda introduced by General Bonaparte.

I cambiamenti politici in Spagna dopo Brumaio. L'avvenimento di Brumaio fu ben accolto negli ambienti ufficiali spagnoli. La corte di carlo IV si aspettava dal nuovo regime francese l'aiuto necessario per realizzare i suoi progetti di politica estera, ma anche qualcosa di più importante: la fine della Rivoluzione in Francia e, di conseguenza, la fine dei tentativi di esportarla in Spagna. Questo modo di interpretare il repentino cambiamento politico della francia creo un nuovo cli di opposizione al governo di Mariano L. Urquijo, che si era impegnato in un'ambiziosa politica riformista, ma che nel dicembre $1800 \mathrm{fu}$ obbligato a dare le dimissioni. Contrariamente a cio che si era verificato all'epoca del direttorio, non ci fu un diretto intervento 
francese sugli affari interni spagnoli, ma in politica estera l'influenza di Bonaparte fu diretta e determinante e creo nuove incertezze sulla posizione internazionale della Spagna, rendendo più diff $\sim$ cili le sue relazioni con la Francia e accrescendo il suo impegno internazionale. La monarchia spagnola accordo eccessiva fiducia al nuovo regime francese e fu incapace di adattarsi o di opporsi ale nuove pratiche introdotte dal generale Bonaparte.

Cambios políticos en Espagña después de Brumario. Los medios oficiales españoles acogieron de forma positiva la noticia del golpe de Estado de Brumario. La corte española esperó recibir del nuevo régimen establecido en Francia la ayuda necesaria para cumplir los proyectos exteriores de la monarquía y, asimismo, tuvo la esperanza de que finalizara la revolución, lo cual implicaba el cese de las tentativas para exportarla a España. Esta forma de interpretar el cambio político francés creó un ambiente contrario al gobierno dirigido por Mariano L. de Urquijo, comprometido en una ambiciosa política reformista, y en diciembre de 1800 Urquijo fue obligado a dimitir de su cargo. Al contrario de lo que sucediera en la época del Directorio, el Consulado no intervino directamente en el primer momento en los asuntos internos españoles, pero en política exterior resultó determinante la influencia de Bonaparte, la cual creó nuevas incertidumbres acerca de la posición internacional de España, incrementando su compromiso internacional y complicando sus relaciones con Francia. La monarquía española adoleció de excesiva confianza en el nuevo régimen frances y se manifestó incapaz de adaptarse o de oponerse a los nuevos usos introducidos por el general Bonaparte.

Die politischen Änderungen nach Brumaire in Spanien. Der Staatsstreich vom Brumaire wurde in den spanischen offiziellen Kreisen gut aufgenommen. Der Hof von Carlos IV. erwartete vom neuen französischen Regime die nötige Hilfe, um seine außenpolitischen Pläne zu verwirklichen, aber auch etwas Wichtigeres, nämlich das Ende der Revolution und damit das Ende der Versuche, diese nach Spanien einzuführen. Diese Art, den politischen Wechsel in Frankreich zu interpretieren, schuf ein neues Klima, das der reformfreudigen, von Mariano L. Urquijo geleiteten Regierung entgegenstand. Im Dezember 1800 mußte er sein Amt niederlegen. Im Gegensatz zu der vom Direktorium durchgeführten Politik versuchte nun Frankreich nicht mehr, in die inneren Angelegenheiten Spaniens einzugreifen, aber auf dem außenpolitischen Gebiet war Bonapartes Einfluß unmittelbar und ausschlaggebend. Dies führte in Spaniens internationaler Stellung $\mathrm{zu}$ neuen Ungewißheiten, die seine Beziehungen $\mathrm{zu}$ Frankreich erschwerten und seine internationalen Verpflichtungen vergrößerten. Die spanische Monarchie vertraute zu sehr der neuen französischen Regierung und war unfähig, sich den neuen, von General Bonaparte eingeführten Bräuchen anzupassen oder entgegenzustellen.

\section{AUTEUR}

\section{EMILIO LA PARRA LÓPEZ}

Université d'Alicante 